\title{
REPRODUCING KERNELS FOR $q$-JACOBI POLYNOMIALS ${ }^{1}$
}

\author{
WALEED A. AL-SALAM AND MOURAD E. H. ISMAIL
}

\begin{abstract}
We derive a family of reproducing kernels for the $q$-Jacobi polynomials $\Phi_{n}^{(\alpha, \beta)}(x)={ }_{2} \Phi_{1}\left(q^{-n}, q^{n-1+\beta} ; q^{\alpha} ; q, q x\right)$. This is achieved by proving that the polynomials $\Phi_{n}^{(\alpha, \beta)}(x)$ satisfy a discrete Fredholm integral equation of the second kind with a positive symmetric kernel, then applying Mercer's theorem.
\end{abstract}

1. Introduction. The purpose of the present note is to construct a family of reproducing kernels or bilinear formulas

$$
\sum_{n=0}^{\infty} \theta_{n}^{(j)} \Phi_{n}^{(\alpha, \beta)}(x) \Phi_{n}^{(\alpha, \beta)}(y)=K^{(j)}(x, y)
$$

for the $q$-Jacobi polynomials $\left\{\Phi_{n}^{(\alpha, \beta)}(x)\right\}_{n=0}^{\infty}$. For definitions and notations, see $\$ 2$. These reproducing kernels are obtained by finding a linear integral operator that maps a $q$-Jacobi polynomial to another $q$-Jacobi polynomial of the same degree but with different parameters. This integral operator is the $q$-fractional integral $L^{(\alpha, \eta)}$ of (2.9). The results obtained below are $q$-analogues of Ismail's results in [5]. Actually Al-Salam and Ismail [1] used certain discrete transforms, that map the Charlier and Meixner polynomials to Laguerre polynomials, to derive several bilinear formulas for the Charlier and Meixner polynomials. Later Ismail [5] modified these transforms and obtained similar formulas for the Hahn polynomials. Related results were also obtained by Rahman [6], [7] by using a completely different approach.

In the next section we define the $q$-Jacobi polynomials and a $q$-fractional integral operator. $\$ 3$ contains our main results, the family of bilinear formulas (3.13). The last section, $\S 4$, is devoted to proving the square integrability of the kernel $K\left(q^{r}, q^{s} ; \alpha, \beta, \eta ; q\right)$ of (3.6) with respect to the measure $\mu(x, y)$ of (3.9) and (3.10).

For an excellent survey of the theory of reproducing kernels we refer the interested reader to Hille [4].

2. Preliminaries. Throughout this work we shall always assume that $0<q$ $<1$. The symbol $(a ; q)_{\infty}$ shall stand for the convergent infinite product

Received by the editors February 25, 1977.

AMS (MOS) subject classifications (1970). Primary 33A65, 33A70; Secondary 33A30.

Key words and phrases. $q$-Jacobi polynomials, $q$-Laguerre polynomials, connection relations, bilinear forms, reproducing kernels, discrete integral equations, Mercer's theorem, symmetric kernels.

'This research was supported by the National Research Council of Canada. 
$\Pi_{0}^{\infty}\left(1-a q^{n}\right)$. By $(a ; q)_{\alpha}$, or equivalently $[1-a]_{\alpha}$ we mean

$$
(a ; q)_{\alpha}=[1-a]_{\alpha}=(a ; q)_{\infty} /\left(a q^{\alpha} ; q\right)_{\infty},
$$

so that, in particular, we have $(a ; q)_{0}=1,(a ; q)_{n}=(1-a)(1-a q) \cdots(1$ $-a q^{n-1}$ ) for $n=1,2,3, \cdots$.

The generalized basic or $q$-hypergeometric function ${ }_{r} \Phi_{s}$ is defined by

$$
\begin{aligned}
{ }_{r} \Phi_{s}\left(a_{1}, \ldots,\right. & \left.a_{r} ; b_{1}, \ldots, b_{s} ; q, z\right) \\
& ={ }_{r} \Phi_{s}\left[\begin{array}{l}
a_{1}, a_{2}, \ldots, a_{r} ; \\
b_{1}, b_{2}, \ldots, b_{s} ;
\end{array}\right] \\
& =\sum_{n=0}^{\infty} \frac{\left(a_{1} ; q\right)_{n}\left(a_{2} ; q\right)_{n} \cdots\left(a_{r} ; q\right)_{n}}{\left(b_{1} ; q\right)_{n}\left(b_{2} ; q\right)_{n} \cdots\left(b_{s} ; q\right)_{n}} \frac{z^{n}}{(q ; q)_{n}} .
\end{aligned}
$$

In particular, we have the $q$-analogue of the binomial theorem [8, p. 92]

$$
{ }_{1} \Phi_{0}(a ;-; q, z)=(a z ; q)_{\infty} /(z ; q)_{\infty}
$$

valid for $|z|<1$.

Let us recall the $q$-integral

$$
\int_{0}^{x} f(t) d(t ; q)=x(1-q) \sum_{n=0}^{\infty} q^{n} f\left(x q^{n}\right) .
$$

This note is concerned with what we call $q$-Jacobi polynomials as defined by

$$
\Phi_{n}^{(\alpha, \beta)}(x)={ }_{2} \Phi_{1}\left(q^{-n}, q^{n+\beta-1} ; q^{\alpha} ; q ; q x\right) .
$$

However we should mention here that Andrews and Askey [2] studied a more general set of polynomials, namely, ${ }_{3} \Phi_{2}\left(q^{-n}, q^{n+\alpha+\beta+1}, x ; q^{\alpha+1},-q^{d+1}\right.$; $q, q)$, which they also refer to as the $q$-Jacobi polynomial. They refer to (2.4) as the ${ }_{2} \Phi_{1}$-Jacobi polynomials.

The $q$-Jacobi polynomials satisfy the orthogonality relation

$$
\int_{-\infty}^{\infty} \Phi_{n}^{(\alpha, \beta)}(x) \Phi_{m}^{(\alpha, \beta)}(x) d \Psi(x ; \alpha, \beta)=F_{n}(\alpha, \beta) \delta_{n m}
$$

where

$$
F_{n}(\alpha, \beta)=q^{\alpha n} \frac{(q ; q)_{n}\left(q^{\beta-\alpha} ; q\right)_{n}\left(q^{n+\beta-1} ; q\right)_{n}}{\left(q^{\alpha} ; q\right)_{n}\left(q^{\beta} ; q\right)_{2 n}}
$$

and $\Psi(x ; \alpha, \beta)$ is the step function with the jumps

$$
d \Psi(x ; \alpha, \beta)=\frac{\left(q^{\alpha} ; q\right)_{\infty}\left(q^{\beta-\alpha} ; q\right)_{k}}{\left(q^{\beta} ; q\right)_{\infty}(q ; q)_{k}} q^{\alpha k}
$$

at $x=q^{k}(k=0,1,2,3, \ldots), \beta>\alpha>0$.

(2.5) may also be written as a $q$-integral 


$$
\begin{array}{r}
\int_{0}^{1}[1-q t]_{\beta-\alpha-1} t^{\alpha-1} \Phi_{n}^{(\alpha, \beta)}(t) \Phi_{m}^{(\alpha, \beta)}(t) d(t ; q) \\
=\frac{(q ; q)_{\infty}\left(q^{\beta} ; q\right)_{\infty}}{\left(q^{\beta-\alpha} ; q\right)_{\infty}\left(q^{\alpha} ; q\right)_{\infty}} F_{n}(\alpha, \beta) \delta_{n m}
\end{array}
$$

We shall also make use of the fractional operator $\mathfrak{L}^{(\alpha, \eta)}$ for $\eta>0$,

$$
\begin{aligned}
& \mathcal{L}^{(\alpha, \eta)} f(x)=\int_{0}^{1} f(x t) d \Psi(t ; \alpha, \eta+\alpha) \\
& \quad=\frac{\left(q^{\alpha} ; q\right)_{\infty}\left(q^{\eta} ; q\right)_{\infty}}{\left(q^{\alpha+\eta} ; q\right)_{\infty}(q ; q)_{\infty}(1-q)} \cdot \int_{0}^{1}[1-q t]_{\eta-1} t^{\alpha-1} f(x t) d(t ; q) .
\end{aligned}
$$

In particular, using (2.2), we can show that

$$
\mathcal{L}^{(\alpha, \eta)}\left\{x^{n}\right\}=\frac{\left(q^{\alpha} ; q\right)_{n}}{\left(q^{\alpha+\eta} ; q\right)_{n}} x^{n} \quad(n=0,1,2,3, \cdots)
$$

so that

$$
\mathfrak{L}^{(\alpha, \eta)} \Phi_{n}^{(\alpha, \beta)}(x)=\Phi_{n}^{(\alpha+\eta, \beta)}(x) .
$$

More explicitly, (2.11) can also be written as

$$
\sum_{k=0}^{\infty} q^{\alpha k} \frac{\left(q^{\alpha} ; q\right)_{\infty}\left(q^{\eta} ; q\right)_{k}}{\left(q^{\eta+\alpha} ; q\right)_{\infty}(q ; q)_{k}} \Phi_{n}^{(\alpha, \beta)}\left(x q^{k}\right)=\Phi_{n}^{(\alpha+\eta, \beta)}(x) .
$$

3. Reproducing kernels. (2.11) shows that the $q$-fractional integral operator $\mathcal{L}^{(\alpha, \beta)}$ maps a $q$-Jacobi polynomial to a $q$-Jacobi polynomial. Hence we have

$$
\begin{aligned}
F_{n}(\alpha+ & \eta, \beta) \delta_{n m} \\
& =\int_{-\infty}^{\infty}\left\{\varrho^{(\alpha, \eta)} \Phi_{n}^{(\alpha, \beta)}(x)\right\}\left\{\varrho^{(\alpha, \eta)} \Phi_{n}^{(\alpha, \beta)}(x)\right\} d \Psi(x ; \alpha+\eta, \beta) .
\end{aligned}
$$

Substituting from (2.12) we get

$$
\begin{aligned}
F_{n}(\alpha+\eta ; \beta) \delta_{m n}= & {\left[\frac{\left(q^{\alpha} ; q\right)_{\infty}}{\left(q^{\alpha+\eta} ; q\right)_{\infty}}\right]^{2} \int_{-\infty}^{\infty} \sum_{k, j=0}^{\infty} \frac{\left(q^{\eta} ; q\right)_{j}\left(q^{\eta} ; q\right)_{k}}{(q ; q)_{j}(q ; q)_{k}} } \\
& \cdot q^{\alpha(k+j)} \Phi_{n}^{(\alpha, \beta)}\left(x q^{k}\right) \Phi_{m}^{(\alpha, \beta)}\left(x q^{j}\right) d \Psi(x ; \alpha+\eta, \beta) .
\end{aligned}
$$

Substituting for $d \Psi(x ; \alpha+\eta, \beta)$ in (3.2) from (2.7) we get, after some simplification,

$$
\begin{aligned}
F_{n}(\alpha+\eta ; \beta) \delta_{m n}=( & \left.q^{\alpha} ; q\right)_{\infty}\left(q^{\alpha} ; q\right)_{\infty} /\left(q^{\beta} ; q\right)_{\infty}\left(q^{\alpha+\eta} ; q\right)_{\infty} \\
& \cdot \sum_{r, s=0}^{\infty} \Phi_{n}^{(\alpha, \beta)}\left(q^{r}\right) \Phi_{m}^{(\alpha, \beta)}\left(q^{s}\right) \\
& \cdot q^{\alpha(r+s)} \frac{\left(q^{\eta} ; q\right)_{r}\left(q^{\eta} ; q\right)_{s}}{(q ; q)_{r}(q ; q)_{s}} \\
& \cdot \Phi_{2}\left(q^{-r}, q^{-s}, q^{\beta-\alpha-\eta} ; q^{1-\eta-r}, q^{1-\eta-s} ; q^{2-\eta-\alpha}\right) .
\end{aligned}
$$

Now using (2.6) and (3.3) we obtain 
$F_{n}(\alpha ; \beta) \delta_{m n}=\frac{\left(q^{\alpha} ; q\right)_{\infty}\left(q^{\beta-\alpha} ; q\right)_{n}\left(q^{\alpha+\eta} ; q\right)_{n}}{\left(q^{\alpha+\eta} ; q\right)_{\infty}\left(q^{\beta-\alpha-\eta} ; q\right)_{n}\left(q^{\alpha} ; q\right)_{n}}$

$$
\begin{aligned}
& \cdot \sum_{r=0}^{\infty} \Phi_{n}^{(\alpha, \beta)}\left(q^{r}\right) \frac{\left(q^{\eta} ; q\right)_{r}}{\left(q^{\beta-\alpha} ; q\right)_{r}} \sum_{s=0}^{\infty} \Phi_{m}^{(\alpha, \beta)}\left(q^{s}\right) \frac{\left(q^{\eta} ; q\right)_{s}}{(q ; q)_{s}} q^{\alpha s} \\
& { }_{3} \Phi_{2}\left(q^{-r}, q^{-s}, q^{\beta-\alpha-\eta} ; q^{1-\eta-r}, q^{1-\eta-s} ; q, q^{2-\eta-\alpha}\right) d \Psi\left(q^{r} ; \alpha, \beta\right) .
\end{aligned}
$$

Combining (3.4) and (2.5) with the uniqueness of the orthogonal polynomials we get

$$
\begin{aligned}
\Phi_{n}^{(\alpha, \beta)}\left(q^{r}\right)= & \frac{\left(q^{\alpha} ; q\right)_{\infty}\left(q^{\beta-\alpha} ; q\right)_{n}\left(q^{\alpha+\eta} ; q\right)_{n}}{\left(q^{\alpha+\eta} ; q\right)_{\infty}\left(q^{\beta-\alpha-\eta} ; q\right)_{n}\left(q^{\alpha} ; q\right)_{n}} \frac{\left(q^{\eta}, q\right)_{r}}{\left(q^{\beta-\alpha} ; q\right)_{r}} q^{-n \eta} \\
& \left\{\sum_{s=0}^{\infty} \Phi_{n}^{(\alpha, \beta)}\left(q^{s}\right) q^{\alpha s} \frac{\left(q^{\eta} ; q\right)_{s}}{(q ; q)_{s}}\right. \\
& \left.{ }_{3} \Phi_{2}\left(q^{-r}, q^{-s}, q^{\beta-\alpha-\eta} ; q^{1-\eta-r}, q^{1-\eta-s} ; q, q^{2-\eta-\alpha}\right)\right\} .
\end{aligned}
$$

Clearly (3.5) is an integral equation satisfied by $\Phi_{n}^{(\alpha, \beta)}(x)$. The kernel in (3.5) is not symmetric but can be symmetrized by rewriting (3.5) as

$$
\begin{aligned}
q^{\alpha r / 2}\left\{\left(q^{\beta-\alpha} ; q\right)_{r}\left(q^{\alpha} ; q\right)_{\infty} /(q ; q)_{r}\left(q^{\beta} ; q\right)_{\infty}\right\}^{1 / 2} \Phi_{n}^{(\alpha, \beta)}\left(q^{r}\right) \\
=\lambda_{n}(\alpha, \beta, \eta, q) \sum_{s=0}^{\infty} q^{\alpha s / 2}\left\{\frac{\left(q^{\beta-\alpha} ; q\right)_{s}\left(q^{\alpha} ; q\right)_{\infty}}{(q ; q)_{s}\left(q^{\beta}, q\right)_{\infty}}\right\}^{1 / 2} \\
\Phi_{n}^{(\alpha, \beta)}\left(q^{s}\right) K\left(q^{s}, q^{r} ; \alpha, \beta, \eta, q\right) .
\end{aligned}
$$

where $K\left(q^{s}, q^{r} ; \alpha, \beta, \eta, q\right)$ is the symmetric kernel

$K\left(q^{s}, q^{r} ; \alpha, \beta, \eta ; q\right)=q^{(r+s) \alpha / 2}\left(q^{\eta} ; q\right)_{r}\left(q^{\eta} ; q\right)_{s}$

$$
\begin{aligned}
\cdot & \left\{\left(q^{\beta-\alpha} ; q\right)_{r}\left(q^{\beta-\alpha} ; q\right)_{s}(q ; q)_{r}(q ; q)_{s}\right\}^{-1 / 2} \\
& { }_{3} \Phi_{2}\left(q^{-r}, q^{-s}, q^{\beta-\alpha-\eta} ; q^{1-\eta-r}, q^{1-\eta-s} ; q, q^{2-\eta-\alpha}\right),
\end{aligned}
$$

and $\lambda_{n}$ are the eigenvalues

$$
\lambda_{n}(\alpha, \beta, \eta ; q)=\frac{\left(q^{\alpha+\eta} ; q\right)_{\infty}\left(q^{\beta-\alpha} ; q\right)_{n} q^{-n \eta}}{\left(q^{\alpha+\eta+n} ; q\right)_{\infty}\left(q^{\beta-\alpha-\eta} ; q\right)_{n}} \quad(n=0,1,2, \ldots) .
$$

The completeness of the system of orthogonal polynomials $\left\{\Phi_{n}^{(\alpha, \beta)}(x)\right\}_{n=0}^{\infty}$ follows from Weierstrass' approximation theorem. Therefore, the eigenvalues given by (3.7) are all the eigenvalues of the integral equation

$$
f\left(q^{r}\right)=\lambda \sum_{s=0}^{\infty} K\left(q^{r}, q^{s} ; \alpha, \beta, \eta ; q\right) f\left(q^{s}\right), \quad r=0,1,2, \ldots
$$

The eigenvalues $\lambda_{n}(\alpha, \beta, \eta ; q)$ are positive and increasing to $\infty$ for $\alpha>0$, 
$\beta>\alpha+\eta$ and $\eta>0$, and the symmetric kernel $K\left(q^{r}, q^{s} ; \alpha, \beta, \eta ; q\right)$ belongs to $L^{2}(d \mu)$ where

$$
\mu(x, y)=\nu(x) \nu(y)
$$

and

$$
d v(x)= \begin{cases}1 & \text { if } x=q^{k}, k=0,1, \ldots, \\ 0 & \text { otherwise }\end{cases}
$$

see $\S 4$. Now we are in a position to apply the extension of Mercer's theorem ${ }^{2}$ (see Tricomi $[9$, p. 125]) to the discrete integral equation (3.8) and derive the bilinear formula

$$
\sum_{n=0}^{\infty} \frac{f_{n}\left(q^{r} ; \alpha, \beta\right) f_{n}\left(q^{s} ; \alpha, \beta\right)}{\lambda_{n}(\alpha, \beta, \eta ; q)}=K\left(q^{r}, q^{s} ; \alpha, \beta, \eta ; q\right)
$$

with

$$
f_{n}\left(q^{r} ; \alpha, \beta\right)=q^{\alpha r / 2}\left\{\frac{\left(q^{\beta-\alpha} ; q\right)_{r}\left(q^{\alpha} ; q\right)_{\infty}}{(q ; q)_{r}\left(q^{\beta} ; q\right)_{\infty}}\right\}^{1 / 2} \Phi_{n}^{(\alpha, \beta)}\left(q^{r}\right) .
$$

The reproducing kernel (3.6) can be iterated to give

$$
\begin{aligned}
& \sum_{n=0}^{\infty}\left\{\lambda_{n}(\alpha, \beta, \eta ; q)\right\}^{-j} f_{n}\left(q^{r} ; \alpha, \beta\right) f_{n}\left(q^{s} ; \alpha, \beta\right) \\
& =K^{(j)}\left(q^{r}, q^{s} ; \alpha, \beta, \eta ; q\right), \quad j=1,2, \ldots,
\end{aligned}
$$

and the iterated kernels $K^{(j)}$ are defined inductively by

$$
\begin{aligned}
& K^{(1)}\left(q^{r}, q^{w} ; \alpha, \beta, \eta ; q\right)=K\left(q^{r}, q^{s} ; \alpha, \beta ; \eta ; q\right), \\
& K^{(j+1)}\left(q^{r}, q^{s} ; \alpha, \beta, \eta ; q\right) \\
& \quad=\sum_{l=0}^{\infty} K^{(j)}\left(q^{r}, q^{l} ; \alpha, \beta, \eta ; q\right) K\left(q^{l}, q^{s} ; \alpha, \beta, \eta ; q\right) .
\end{aligned}
$$

One can easily take special cases of the above bilinear formulas and derive similar formulas for the $q$-Laguerre and $q$-Legendre polynomials.

4. The square integrability of $K\left(q^{r}, q^{s} ; \alpha, \beta, \eta ; q\right)$. In this section we shall prove that the kernel $K\left(q^{r}, q^{s} ; \alpha, \beta, \eta ; q\right)$ of (3.6) belongs to $L^{2}(d \mu), \mu$ being the measure defined by (3.4) and (3.10). Let $\|K\|_{\mu}$ be the $L^{2}(d \mu)$ norm of the above mentioned kernel. We now prove that $\|K\|_{\mu}$ is finite.

From (3.5) we have, for $\alpha>0, \beta>\alpha+\eta, \eta>0$,

$$
\begin{aligned}
\|K\|_{\mu}^{2}=\sum_{r, s=}^{\infty} \frac{\left(q^{\eta} ; q\right)_{r}\left(q^{\eta} ; q\right)_{s} q^{\alpha(r+s)}}{\left(q^{\beta-\alpha} ; q\right)_{r}\left(q^{\beta-\alpha} ; q\right)_{s}(q ; q)_{r}(q ; q)_{s}} \\
\cdot \cdot\left\{{ }_{3} \Phi_{2}\left(q^{-r}, q^{-s}, q^{\beta-\alpha-\eta} ; q^{1-\eta-r}, q^{1-\eta-s} ; q, q^{2-\eta-\alpha}\right)\right\}^{2} .
\end{aligned}
$$

${ }^{2}$ The extension is straightforward and we believe it is known. 
Applying the Cauchy-Schwarz inequality on the $\left\{{ }_{3} \Phi_{2}\right\}^{2}$ we get

$$
\begin{aligned}
\|K\|_{\mu}^{2} \leqslant & \sum_{r, s=0}^{\infty} \frac{\left(q^{\eta} ; q\right)_{r}^{2}\left(q^{\eta} ; q\right)_{s}^{2} q^{\alpha(r+s)}}{\left(q^{\beta-\alpha} ; q\right)_{r}\left(q^{\beta-\alpha} ; q\right)_{s}(q ; q)_{r}(q ; q)_{s}} \\
& \cdot \sum_{k} r s \frac{\left(q^{-r} ; q\right)_{k}^{2}\left(q^{-s} ; q\right)_{k}^{2}\left(q^{\beta-\alpha-\eta} ; q\right)_{k}^{2}}{(q ; q)_{k}^{2}\left(q^{1-\eta-r} ; q\right)_{k}^{2}\left(q^{1-\eta-s} ; q\right)_{k}^{2}} q^{2(2-\eta-\alpha) k} .
\end{aligned}
$$

Interchanging summations and using the identity

$$
\left(q^{a-r} ; q\right)_{k}=(-1)^{k} q^{k(a-r)+k(k-1) / 2} \frac{\left(q^{1-a} ; q\right)_{r}}{\left(q^{1-a} ; q\right)_{r-k}}
$$

we get

$$
\|K\|_{\mu}^{2} \leqslant \sum_{k=0}^{\infty} \frac{\left(q^{\beta-\alpha-\eta} ; q\right)_{k}^{2} q^{2 \eta k}}{\left(q^{\beta-\alpha} ; q\right)_{k}}\left\{\sum_{r} \frac{(r+k) q^{\alpha r}\left(q^{1+r} ; q\right)_{r}\left(q^{\eta} ; q\right)_{r}^{2}}{\left(q^{\beta-\alpha+k} ; q\right)_{r}(q ; q)_{r}^{2}}\right\}^{2} .
$$

The inside summation on the right-hand side of the above inequality behaves for large values of $k$ as $(A+B k)^{2}$ where $A$ and $B$ are constants. Hence the right-hand side is convergent (by the ratio test) under the stated conditions. This proves our assertion.

\section{BIBLIOGRAPHY}

1. W. Al-Salam and M. E. H. Ismail, Polynomials orthogonal with respect to a discrete convolution, J. Math. Anal. Appl. 55 (1976), 125-139.

2. G. Andrews and R. Askey, The classical and discrete orthogonal polynomials and their q-analogues (in preparation).

3. W. Hahn, Uber orthogonalpolynome, die q-differenzengleichungen, Math. Nachr. 2 (1949), 4-34.

4. E. Hille, Introduction to general theory of reproducing kernels, Rocky Mountain J. Math. 2 (1972), 321-368.

5. M. E. H. Ismail, Connection relations and bilinear formulas for the classical orthogonal polynomials, J. Math. Anal. Appl. 57 (1977), 487-496.

6. M. Rahman, A five parameter family of positive kernels from Jacobi polynomials, SIAM J. Math. Anal. 7 (1976), 386-413.

7. 7 (1976), 414-435.

8. L. J. Slater, Generalized hypergeometric function, Cambridge Univ. Press, Cambridge, 1966.

9. F. G. Tricomi, Integral equations, Interscience, New York, 1957.

Department of Mathematics, University of Alberta, Edmonton, Alberta, Canada

Department of Applied Mathematics, McMaster University, Hamilton, Ontario, CANADA 